\title{
Comparisons of models of associative recall
}

\author{
BRIAN H. ROSS and GORDON H. BOWER \\ Stanford University, Stanford, California 94305
}

\begin{abstract}
We tested three mathematical models of associative recall that differ in the assumed structure of the memory trace (direct or indirect connections between parts) and the interdependence of the connections (independent or correlated). The three models compared are the "horizontal" model with direct, independent connections, the "schema" model with indirect, independent connections, and the "fragment" model with indirect, correlated connections. In Experiment 1, subjects studied quartets of minimally related words and then recalled, using one or two words of each quartet as cues. The horizontal and schema models predicted the data very well, but the fragment model did poorly. In Experiment 2, related word quintets were learned, and recall cues contained one, two, or three words of a quintet. Only the schema model adequately fit these data. Experiment 3 directly tested the fragment model, using sequential tests of unrelated word quartets. Even an augmented fragment model with extra recall failure parameters was decisively rejected by these data. Both the horizontal and schema models gave satisfactory qualitative accounts, although poor quantitative fits. A means of deriving the models through a spreading activation semantic network of preexperimental knowledge is suggested, and related models are discussed.
\end{abstract}

A fundamental issue in memory theory concerns the structure of the memory trace. The issue is simply this: If one were to draw a diagram to represent the connectivity among distinguishable parts of a memory trace, what would it look like? (The parts may be individual items, features, properties, or objects.) Historically, this issue has rarely been addressed directly; rather, when considered at all, a particular hypothesis was adopted as "natural," and most theorists accepted that view without question.

The traditional view comes from philosophical associationism. It claims that different parts of a memory trace are connected directly to one another (each to every other) by symmetric, bidirectional associations. Except for scattered grumblings from Gestalt psychologists, this particular representation of the memory trace has long been dominant, perhaps because psychologists did not notice that alternative views were possible. In the past 15 years, with the advent of computer simulation models of episodic memory such as HAM (J. R. Anderson \& Bower, 1973) or ELINOR (Norman, Rumelhart, \& the LNR Research Group, 1975), it became apparent that there are alternative representations of the memory trace and that the representation chosen has important consequences for one's theory. With this awareness of alternatives, some mathematical analyses and experimental tests were developed to test a few alternatives (see, e.g., J. R. Anderson \& Bower, 1973, chap. 10; Jones, 1976, 1978a, 1978b), but these tests have not been definitive.

We thank L. Barsalou for comments on an earlier version of the manuscript. This research was supported by Grant MH13950 from the National Institutes of Mental Health to G. H. Bower. Address correspondence to the authors at the Department of Psychology, Stanford University, Stanford, California 94305.
Before discussing experimental tests, however, we will first consider the range of alternative models of the memory trace within a larger framework. At the outset, we note that the basic facts of memory to be explained concern the way that different parts of an input event later act as cues that can retrieve from memory other parts of the initial event or its experience. For a cluster of distinguishable parts of an event, the memory trace summarizes a set of interrelated cue-to-output retrieval functions. For this reason, most memory theories represent these cue-response retrieval routes directly in the trace by supposing that (1) parts of the event are mapped directly into parts of the trace, and (2) retrieval routes from cue to response are mapped into some kind of connection or link (direct or indirect) between those two parts in the trace. (Perhaps even distributed memories, such as those proposed by J. A. Anderson (1977) and Kohonen (1977), can be cast into such a representation.) The connections or links between parts, or groups of parts, of the trace will be called by the familiar label "associations."

Within this associative framework, specific trace models differ in at least two ways. First, models may differ in the way the parts of the trace are presumed to be connected together (for illustrations, see Figure 1). For example, the aforementioned associationist doctrine claimed that each part of a trace could be connected by a single direct linkage to any other part. However, an alternative assumption is that parts of a trace may be connected to an abstract, higher order node representing the trace as a whole and that parts are only indirectly connected to other parts by virtue of their individual connections to the common trace node. We will later consider versions of each of these trace representations.

A second distinguishing feature of trace models is whether the associations connecting parts are presumed 
to be unidirectional (asymmetric) or bidirectional (symmetric). If a theory supposes that an association exists from Part (Node) A to Part (Node) B, it may or may not be committed to the existence of a correlated association in the opposite direction, from B to $\mathrm{A}$. This issue surfaced in the experimental debate over associative symmetry (e.g., Asch \& Ebenholtz, 1962), asking whether the so-called "forward" and "backward" links in paired associate learning were distinct or correlated. For example, Wolford (1971) and others found that the forward and backward links were not perfectly correlated, suggesting somewhat independent links. This issue of correlated vs. independent links between two nodes can in fact be generalized to all links in a larger associative structure. Thus, "all-or-none" memory models for propositions (e.g., R. C. Anderson, 1977; Goetz, Anderson, \& Schallert, Note 1) assume that for suitably unitary structures (atomic propositions) all links within the trace are either established together or none are established at all as the result of one study trial with the to-be-learned material. In contrast, other models like HAM (J. R. Anderson \& Bower, 1973) assume that the links among units within a single trace are established with independent probabilities as the result of a single study trial, although this learning probability may vary across subjects and materials.

In this paper, we examine three specific trace models that have received recent attention. The first two models assume independently established unidirectional associations. In one case, the associations directly link elements of the event; in the other case, the associations among elements are indirect, mediated through an abstract node common to the trace of the event. The third model is the "memory fragment" model of Jones (1976), which differs from the first two in associative structure and in the assumed interdependence of its links. Each theory can be formulated as a mathematical model, the details of which will be tested against experimental results.

The following discussion of competing models will refer to the learning task used in our first experiment. Our subjects studied unrelated clusters of four nouns for one trial, and then received one or two words from each cluster for a cued recall test. The reason we had subjects learning clusters of four words, rather than the traditional "paired associates," is because two of the three models yield identical predicitons for paired associate results. Therefore, larger associative groupings (three or more) are required to obtain results that potentially can be discriminating. Perhaps the traditional paired associate methodology [and the stimulus-response (S-R) model] partly prevented awareness of alternatives to the traditional associative trace model of memory.

The tests involved presenting one or two cues from a cluster and having the subject try to recall the remaining words of that cluster. The one-cue vs. two-cue tests are included to provide more outcomes for the models to predict. For the theories to be tested, recall predictions for one- and two-cue tests involve the same parameters, so the extent of the change with an additional retrieval cue provides an interesting datum for distinguishing predictions of the models. [See Bruce (1980) for a discussion of various combination rules of cues. '] In model testing, all items are presumed to be statistically homogeneous, so that attention will focus on how many items of a cluster are recalled, not which ones are recalled. In the theoretical diagrams, we treat the associations between words or between words and higher order nodes as one-step links. The reader may interpret these as a shorthand for a chain of at least two links: The word is linked to a relevant concept, and a token of that concept is linked to other concepts or to higher order nodes. In Figure 1 and throughout the paper, the probability attached to a link in the diagram may be interpreted as this joint probability that the word as a stimulus succeeds in accessing both its relevant concept and the link from that node to other nodes in the structure. With this background, we now formulate the three competing models to be tested.

\section{Horizontal Model}

The horizontal model assumes that only direct itemto-item associations exist, with directed associations in one or both directions. Panel a of Figure 1 shows this model for a cluster of four nouns with all possible associations depicted. The horizontal model follows in the spirit of S-R learning theory and traditional associationism.

We assume that each directed link in Figure 1a may be available (retained) in memory independently in an all-or-none manner with probability $\theta$ after a standard learning period. With probability $1-\theta$ a link will not be available in memory at the time of the retention test. To make this model comparable in power to its competitors, we had to add a second, encoding process. We assumed that occasionally a learner was unable to incorporate a given word into his or her associative cluster. In Figure 1a the failure to encode a given word would be equivalent to deleting that word from the structure shown. We let $\mathrm{p}$ denote the independent probability that an item is encoded or connected into the trace structure, and $1-p$ denote the probability that it is not so incorporated.

After a single study trial with a single quartet, the subject's memory structure for that quartet at testing consists of a subset of the nodes and links depicted in Figure 1a. The learning assumptions stated above allow us to calculate (as a function of $\theta$ and $p$ ) the likelihood of any possible configuration of accessible nodes and links in the memory trace. The trace configuration available at the time of the cued recall test with a quartet then provides the basis for performance.

To illustrate how the model relates to performance, the probability that one item will cue recall of another item is the likelihood that the cue is effectively connected to the "response" through some properly directed path 
(a)

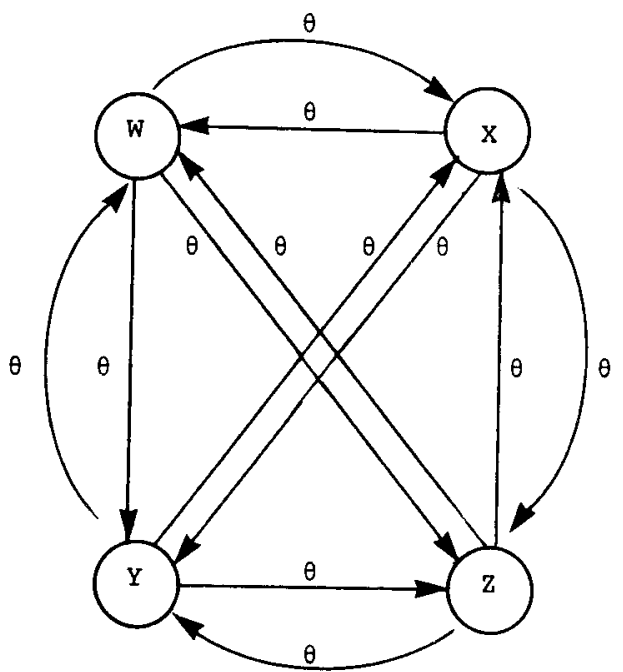

(b)

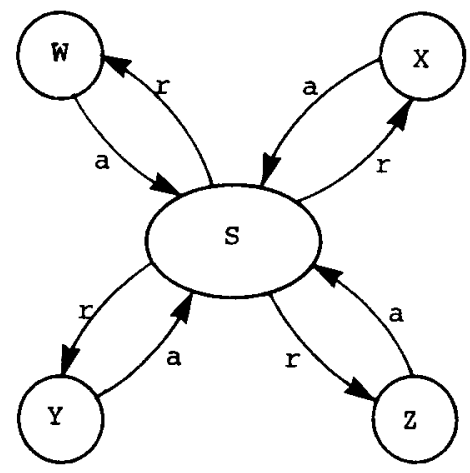

Figure 1. Representation of the (a) horizontal and (b) schema memory structures for a given word quartet.

in the graph structure. Thus, item $\mathrm{X}$ may be recalled to item $\mathrm{W}$ as a cue without a direct $\mathrm{W}$ to $\mathrm{X}$ association if an indirect path can be traced through some intermediaries, such as from $\mathrm{W}$ to $\mathrm{Y}$ and from $\mathrm{Y}$ to $\mathrm{X}$. In fact, there are so many possible connections from any item to every other in Figure 1a that even modest values of $\theta$ can produce very high levels of total recall and practically no total failures of recall. Since both partial recall and total failures of recall are often observed, we added the $\mathrm{p}$ encoding process so that the model's predictions would be closer to the data.

To illustrate the model's calculations, consider its predictions for a one-cue test of a studied quartet. We will calculate the probability that for a randomly selected quartet a random subject will recall exactly two of the three remaining words in such a test. This event can arise from several different trace configurations for a quartet (see Figure 1a). A first possible configuration is that exactly three items were encoded, with the cue being one of them [this has probability $\left..75 p^{3}(1-p)\right]$, and the cue was connected directly to the other two items $\left(\theta^{2}\right)$ or connected directly to one of the other two and then indirectly to the other $\left[2 \theta^{2}(1-\theta)\right]$. Thus, the probability of this event would be $\left[.75 \mathrm{p}^{3}(1-\mathrm{p})\left(\theta^{2}+2 \theta^{2}(1-\theta)\right]\right.$. As a second route, perhaps all four items might have been encoded $\left(\mathrm{p}^{4}\right)$, but no associations were established to some one of the three noncues $\left[3(1-\theta)^{3}\right]$. If so, the two items may be recalled, as with the three-item encoded case above, both directly $\left(\theta^{2}\right)$ or one indirectly $\left[2 \theta^{2}(1-\theta)\right]$. Thus, the probability of this event is $\left[\mathrm{p}^{4} 3(1-\theta)^{3}\right.$ $\left(\theta^{2}+2 \theta^{2}(1-\theta)\right]$. Then the total probability of recalling two items, given one cue, would be the sum of these two routes. Predictions for other possibilities may be calculated in similar fashion. The equations are not included to save space, but are available from the authors.

\section{Schema Model}

The schema model permits only indirect associations among items through a grouping node, labeled $S$ in Panel b of Figure 1. The associations depicted in Figure $1 \mathrm{~b}$ are assumed to be independently established in an all-or-none manner.

In Figure $1 \mathrm{~b}$, the letters $\mathrm{W}, \mathrm{X}, \mathrm{Y}, \mathrm{Z}$ denote the four words of a cluster that is to be learned, and the central hub represents the particular memory schema activated contiguously with the words and within which the words play some part. In the mathematical formulation, each item in the memory trace is characterized by two parameters, a and $r$. Parameter a (for access) is the likelihood that, at the time of retention testing, presentation of that word will allow access to the memory schema for that quartet. We will think of this as an all-or-none affair: At any particular test occasion, a given word cue either fully accesses and activates the correct memory schema or it fails completely. Parameter r (for response) is the likelihood that, during retention testing, a given word will be recallable once the memory schema has been activated.

To relate this model to recall, we let $T$ denote the number of the remaining words of a quartet recalled. The probability distribution of $T$ when the subject is given one word as a cue is as follows:

$\operatorname{Pr}(T=i \mid 1$ cue $)= \begin{cases}1-a+a(1-r)^{3}, & \text { for } i=0 \\ a\left(\begin{array}{l}3 \\ i\end{array}\right) r^{i}(1-r)^{3-i}, & \text { for } i \geqslant 1,\end{cases}$

where

$$
\left(\begin{array}{l}
3 \\
i
\end{array}\right)=\frac{3 !}{i !(3-i) !} \text {. }
$$

To understand some of the terms in Equation 1, we note that the cue word gains access to the schema with probability a; once the schema is accessed, there is independent binomial probability $r$ that each of the 
three responses will be recalled. The probability that the cue leads to no recall $(i=0)$ is the likelihood that it fails to activate the schema, $1-a$, or that it activates the schema but none of the three correct responses can be output, $a(1-r)^{3}$.

A similar equation may be derived for the two-cue tests. The probability of recalling $\mathrm{k}$ remaining words of the quartet given two cues is as follows:

$$
\begin{aligned}
& \operatorname{Pr}(T=k \mid 2 \text { cues })= \\
& \begin{cases}(1-a)^{2}+[a+a(1-a)](1-r)^{2}, & \text { for } k=0 \\
{[a+a(1-a)]\left(\begin{array}{l}
2 \\
k
\end{array}\right) r^{k}(1-r)^{2-k},} & \text { for } k \geqslant 1 .\end{cases}
\end{aligned}
$$

The interpretations of the terms in Equation 2 are as follows. The term $[a+a(1-a)]$ is the probability that one or the other cue word accesses the relevant trace in memory. Once that trace is accessed, the to-berecalled words are output with independent probability, r. This yields the binomial distribution shown, with an additive term at $\mathrm{k}=0$ to reflect the possibility that the memory schema is not accessed at all. (Note that these equations may be generalized to Nelement memory clusters and $\mathrm{J}$ independent cues for recall of the remaining $\mathrm{N}-\mathrm{J}$ items.)

An attractive feature of Equations 1 and 2, as with the horizontal model, is that two-cue results can be related to one-cue results because the same parameters, $a$ and $r$, are involved in both equations. This arises because we have assumed that (1) the words act independently as accessing cues, and (2) once the schema is activated, the subject outputs each word independently with the same probability. The independence assumption is a substantive assumption that is empirically testable. The experiments below permit tests of the independence assumption by checking whether Equations 1 and 2 fit the one-cue and two-cue results.

\section{Fragment Model}

The fragment model, proposed by Jones (1976, 1978a, 1978b), differs from the horizontal and schema model both in terms of associative structure and interdependence of links. According to this model, "the memory trace derived from a particular event corresponds to one or more fragments of the event, each containing a nonoverlapping subset of the components representing that event" (Jones, 1976, pp. 289-290). Cuing with any component of the fragment remaining in memory activates the entire fragment so that the other components of the fragment are produced as responses. The five fragments that might be produced from a quartet (see Figure 2) would be $(4),(3,1),(2,2)$, $(2,1,1)$, and $(1,1,1,1)$. If only a single component of the cluster is included in a fragment, that fragment may be ignored, since it will neither cue nor be cued by any other component. The probabilities of these fragments are not predicted but are to be estimated from the data.

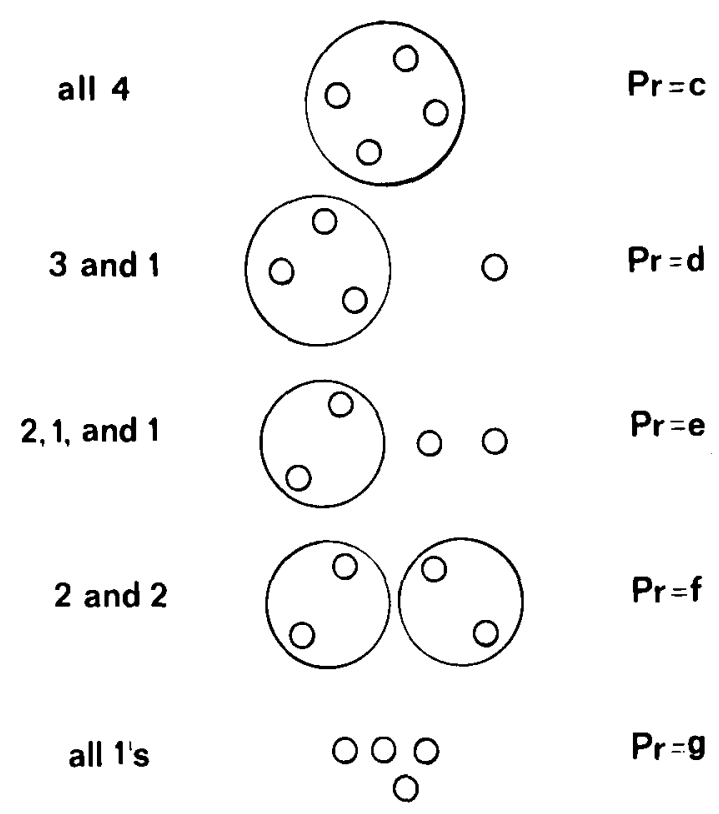

Figure 2. The five logical groupings of four words for the fragment model. The probabilities of the groupings are written to the right.

To illustrate this theory, assume that after studying the quartet $\mathrm{W}, \mathrm{X}, \mathrm{Y}$, and $\mathrm{Z}$, the subject has at the time of the retention test two two-element fragments in memory, namely, $(\mathrm{W}, \mathrm{X})$ and $(\mathrm{Y}, \mathrm{Z})$. [Note that this is only one of the six possible $(2,2)$ fragments.] If cued with $W$, the subject will respond with $X$; if cued with $Y$, the response will be $Z$. If the subject is given two cues, there is an increased probability of recalling a second item, but only if the second cue accesses the second fragment. Thus, in our illustration, a $W$ cue would produce recall of $X$, whereas the $W$ and $Y$ pair of cues would produce recall of $X$ and $Z$. If, instead of a $(2,2)$ fragment, there were a $(3,1)$ fragment of $(W, X, Y)$, then a second cue would improve recall only if the first cue had been $Z$, which did not excite the fragment.

Mathematical formulation of this model is straightforward. Consider one or two items as cues for traces of studied quartets. Figure 2 labels the probability of fragments $(4),(3,1),(2,1,1),(2,2)$, and $(1,1,1,1)$ by $\mathrm{c}, \mathrm{d}, \mathrm{e}, \mathrm{f}$, and $\mathrm{g}$, and these probabilities sum to one. At the time of testing, a subject's memory for a quartet is presumed to be in one of these states, so that all items in the same memory fragment as a cue word will be recalled. Assuming homogeneous selection of the words as members of fragments and as cues, the derived probabilities for the model for one-cue tests are:

$$
\begin{aligned}
& \operatorname{Pr}(\mathrm{T}=0 \mid 1 \text { cue })=.50 \mathrm{e}+.25 \mathrm{~d}+\mathrm{g} \\
& \operatorname{Pr}(\mathrm{T}=1 \mid 1 \text { cue })=.50 \mathrm{e}+\mathrm{f} \\
& \operatorname{Pr}(\mathrm{T}=2 \mid 1 \text { cue })=.75 \mathrm{~d} \\
& \operatorname{Pr}(\mathrm{T}=3 \mid 1 \text { cue })=\mathrm{c} .
\end{aligned}
$$


For two-cue tests, the expressions are:

$$
\begin{aligned}
& \operatorname{Pr}(T=0 \mid 2 \text { cues })=.33 e+.33 f+g \\
& \operatorname{Pr}(T=1 \mid 2 \text { cues })=.50 d+.67 \mathrm{e} \\
& \operatorname{Pr}(T=2 \mid 2 \text { cues })=.50 d+.67 f+c
\end{aligned}
$$

Jones (1976, pp. 290-291) points to three basic properties of this model: symmetry, multiple cuing, and all-or-none processing. Symmetry means that if word $X$ cues recall of word $Y$, then $Y$ should cue recall of $X$. Symmetry holds generally for joint recall probabilities to single cues [e.g., $\operatorname{Pr}($ recall of Word 1 and Word $2 /$ Word 3$)=\operatorname{Pr}($ recall of Word 3 and Word 21 Word 1)]. This follows because these joint recall events will occur, according to the model, only when the memory fragment contains at least Words 1,2 , and 3. The multiple-cuing idea was mentioned above, namely, that additional cues improve recall oniy if they activate further fragments from memory. Finally, all-or-none processing should occur at the level of fragments, although the overall recall will usually not be all or none. Jones $(1976,1978 \mathrm{a}, 1978 \mathrm{~b})$ has fitted his model to recall results for pictures and sentences, and the fits have generally been satisfactory. In a reanalysis of results from J. R. Anderson and Bower (1973), Jones (1978b) claimed that the fragment model fit the data more accurately than did the HAM model. Though Jones may be right in rejecting the specific propositional representation of sentences given by HAM (a view concurred in by J. R. Anderson \& Bower, 1980), it does not follow that the general schema model is incorrect.

In a brief comparison, Jones (1976) found that his fragment model fit some of his results better than did the schema model. However, his conclusion can be faulted because his testing method was inappropriate. In fitting the schema model, Jones used only the group's pooled data, but if there are individual differences in recall, then one should not pool results over subjects to fit the model, because the model's equations ( 1 and 2 ) have nonlinear terms. For example, in our Experiment 2 the schema model fit individuals' recall well, but did very poorly if fit to the pooled data. The model is most properly fit only to the results of individual subjects rather than to group data.

The fragment model makes some strong predictions about the trace structure which have not been definitively tested, despite the experimentation regarding associative symmetry. An important distinguishing feature of the fragment model is that it allows for multiple fragments of a quartet with more than one element [such as the $(2,2)$ fragment mentioned earlier]. The schema model has no way to mimic such trace structures without adding complications. However, the empirically estimated probabilities of such multiple fragments have usually been very small over several experiments, ranging from 0 to .05 . Therefore, the truly distinctive feature of the model seems of very small magnitude. Finally, the frag- ment model has only been fit to experiments in which the materials were arbitrary and unrelated, and the model changes that are necessary in order to account for preexperimental connections among the learning items are not obvious.

\section{EXPERIMENT 1}

This first experiment was undertaken for an entirely different purpose, namely, to assess the influence on learning of arousing an appropriate "frame" or "script" as an integrating mnemonic at the time a cluster of words was studied. In retrospect, the experiment inadvertently provided some very orderly data for testing the recall predictions of the several trace models and is reported for that reason. The learning materials were groups of four words that have slight preexperimental connections to a common concept, frame, or script (see Schank \& Abeison, 1977). Any two of the words did not have mucin connection with one another; however, their connection becomes apparent when they are considered within the context of the script or schema. In that context, the words then refer to objects or properties or that larger script. For example, consider one of our word quartets: grease, curtain, prop, program. Because words have multiple interpretations, the first two words suggest, say, a kitchen scene, but the next two words do not fit at all into kitchen. Rather, they suggest "theater piay." Once that schema is aroused, "grease" (as in "greasepaint") and "curtain" can be given a schema-related interpretation. Consider another example: apron, charr brush, clip. Any two of the words suggest some idiosyncratic scenario, but all four words together can be seen to fit into a "haircut" schema. Apparently, two words like "apron" and "brush" are not associated in isolation; rather, they can be seen as connected mainly within the larger context of getting a haircut.

An implication of the foregoing is that the learning of a cluster of four such words should be easy if the people "understand" the relation among the words, that is, if they activate a schema within which the items have their place. Activating a relevant schema is then equivalent to exciting preexisting interrelations among these words, so that learning consists in strengthening the preexisting associations among the presented items. Contrariwise, if the words do not arouse an appropriate schema, then they will continue to appear unrelated and should be difficult to learn as new clusters. In the jargon of verbal learning, the schema is a "mediator" for learning the words: it differs from the usual verbal-learning mediators insofar as the items subsumed under the script are aiready "interassociated" prior to the subject's entry into the experiment.

In this first experiment, we tried to vary the likelihood that the subjects would infer the appropriate script for the quartet of words. For half the subjects, the word quartets, which were only vaguely related, were presented alone for learning. For the remaining 
subjects, as each word quartet was presented for study, the experimenter also read aloud the name of the script or schema (e.g., "haircut," "theater play") within which the items were interconnected. It was expected that subjects given the script mediators would more readily interassociate the words within a quartet than would subjects not given the mediator. This difference should be reflected in higher learning parameters within the models for the group given the script.

This first experiment, then, had two purposes. One aim was to examine the quantitative predictions of three models of memory trace structure for one-cue and twocue results. The second aim was to investigate how active priming of schema mediators affected memory and changes in learning parameters of the models.

\section{Method}

Design. Two groups of subjects had one study-recall cycle on each of four lists of 20 quartets of words. For one group of subjects, the experimenter named the underlying script (e.g., "haircut") as the word quartet was shown during study; for the other group, he said nothing and they simply studied the quartet. After study of a list, a cued recall test occurred: half the quartets at random were cued for recall by presenting a single word, and half were tested by presenting two words from the quartet. In each case, the person was to recall the remaining words of the cued quartet.

Materials. We constructed 80 quartets of words for this experiment. We began with some scenario (e.g., making a movie, surfing, riding a subway) or object (e.g., a can of beer, a clam, a business office) or event (e.g., July 4th Parade, attack on Pearl Harbor). We then selected unique words that, by themselves were only weakly related to the schema. Some examples will illustrate the quartets: princess, mouth, hold, dial for telephone; bag, fire, penknife, boots for camping; scratch, collar, friend, license for dog; driver, trap, rough, handicap for golf; and glove, ring, bruise, bell for boxing. A given word appeared in only one quartet. Due to the selection procedure, the words within a quartet could not be equated on concreteness or frequency of usage; indeed, most do not appear in the standard norms for concreteness. However, the selection procedure produced quartets that were intuitively related, particularly once the schema was mentioned.

The 80 quartets were divided randomly into four lists of 20 , and each subject studied and was tested on all four lists in a random order. For each list, we constructed eight different recall test lists (one per subject per condition). The eight test lists cued recall of a given quartet in eight different ways: four times with each of the single-word cues and four times with a differently selected pair of the four words as cues. Thus, across subjects within a condition, memory of a given cluster was cued in a statistically balanced fashion. The one or two cue words were printed down the left side of recall sheets given to the subjects after they had studied each list. They wrote their recall of the other two or three remaining words in the cued cluster on a line to the right of the cues.

Procedure. The subjects were fully instructed about the learning task, the quartets to be learned, and the cuing tests of recall. In addition, the informed subjects were told that the four words of a cluster were about some specific object, activity, or event and that they would be told what that was as the four words were presented. They were to call to mind the situation or scenario, to notice how the words fit within that scene, and to use it to memorize the words. Control subjects were simply told to study the word quartets as a group to be remembered, but were told nothing about their common schema.
The words were projected onto a screen before the subjects by an overhead projector. The quartets were presented one at a time for $10 \mathrm{sec}$ each. For informed subjects, the name of the mediating schema was said aloud by the experimenter immediately as each quartet was shown. For recall, the subjects had a booklet with four recall pages for the four lists, separated by blank cover sheets. After studying a quartet list, the subjects turned to its recall page, which they covered with a cardboard mask. Upon signal from the experimenter, they slid the mask down the page one line at a time, exposing (in a $1.25 \times 12.5 \mathrm{~cm}$ slit) the successive word cues. They wrote their recall words to the right of the cues. The signal to move the mask down was given every $15 \mathrm{sec}$. The mask helped to isolate recall testing of each quartet since the subjects could not see other cues or responses as they worked on recalling the cued quartet. A 1-min rest occurred after each test list. The subjects knew that each quartet list would be studied and tested just once.

Subjects. The subjects were 16 Stanford undergraduates participating to fulfill a service requirement for an introductory psychology class. They were tested in small groups of one to four. The groups were assigned in semirandom order to the informed and control conditions, with eight subjects in each group.

\section{Results and Discussion}

Group comparisons. The words recalled for each quartet were noted and counted as correct or incorrect accordingly as they duplicated the sense of the words originally presented. Lenient scoring was used, but the conclusions are not affected by stricter scoring. Each quartet was scored according to the number of responses recalled to the one or two cues. Table 1 shows the complete frequency distributions of the 320 scores for the informed and control groups.

Table 1

Observed Frequencies of Responses Recalled to One or Two Cues for Informed and Control Subjects for Experiment 1 and Predicted Frequencies for the Horizontal, Schema, and Fragment Models

\begin{tabular}{ccccc}
\hline $\begin{array}{c}\text { Number } \\
\text { Recalled }\end{array}$ & Observed & $\begin{array}{c}\text { Hori- } \\
\text { zontal }\end{array}$ & Schema & $\begin{array}{c}\text { Frag- } \\
\text { ment }\end{array}$ \\
\hline \multicolumn{5}{c}{ Informed Group: } \\
0 & 134 & 124 & 130 & 123 \\
1 & 37 & 44 & 39 & 42 \\
2 & 79 & 80 & 80 & 84 \\
3 & 70 & 72 & 71 & 71 \\
& Informed Group: Two-Cue Testing Condition \\
0 & 80 & 83 & 80 & 86 \\
1 & 112 & 110 & 110 & 103 \\
2 & 128 & 127 & 130 & 131 \\
& Control Group: One-Cue Testing Condition & \\
0 & 164 & 145 & 158 & 148 \\
1 & 36 & 48 & 39 & 44 \\
2 & 71 & 75 & 72 & 79 \\
3 & 49 & 52 & 51 & 49 \\
& Control Group: Two-Cue Testing Condition & \\
0 & 100 & 105 & 104 & 112 \\
1 & 111 & 113 & 107 & 100 \\
2 & 109 & 102 & 109 & 108 \\
\hline
\end{tabular}

Note-All predicted frequencies are sums of predictions for individual subjects. 
The observed frequency distributions for informed and control groups do not differ significantly according to a chi-square test $\left[\chi^{2}(6)=10.92, p>.05\right]$. The means were also computed and compared. For the one-cue tests, the average number of words recalled (out of 3) was 1.26 for the informed group and 1.02 for the controls. These do not differ significantly $[\mathrm{t}(14)=1.30$, $\mathrm{p}>.10]$. For the two-cue tests, the average number of words recalled (out of 2) was 1.15 for the informed subjects and 1.03 for the controls, which do not differ significantly. Though the explicit naming of the schema during study increased learning in the predicted direction, the effect was too slight to be significant.

In interviewing the control subjects, it became obvious why the groups did not significantly differ. The four words of a quartet practically always suggested a specific schema for the control subjects; they all reported that most quartets reminded them of some scene or activity. After discovering this fact within studying the first few quartets, the controls knew how the quartets had been constructed, so they searched for single concept mediators to memorize later quartets. The control subjects recalled slightly worse on the first list before they had fully "caught on" to the nature of the quartets, but the difference was not statistically significant. To the extent that control subjects were activating the same memory schema as were informed subjects, the two groups became functionally similar, so their similar recall levels are understandable.

The groups did differ in their intrusion errors. While both groups gave similar totals of intrusions, the informed subjects intruded the schema label more frequently than did controls, with an average of 5.8 vs. 1.6 such intrusions per subject over all 80 tests for informed and controls, respectively. The informed subjects also gave more intrusions that were thematically related to the schema, with totals averaging 14.0 per informed subject vs. 10.5 for control subjects. Fully $71 \%$ of the intrusions for the informed subjects (vs. $47 \%$ for the controls) were either the schema word or thematically related to the schema. Finally, the control subjects gave more intrusions of unrelated words ( 8.0 per subject) than did informed subjects (1.2 per subject). The conclusion from these intrusion data is that informed subjects were somewhat more likely to make errors based on the experimenter's target schema. Though control subjects apparently used a schema, it may not always have been the one given to the informed suojects. Some further differences between the two groups will be discussed in considering the parameter changes between groups for each model.

Independently of the informed vs. control difference, we may ask whether two cues are better than one cue in prompting recall of other words in the quartet. Pooling the informed and control groups, the average probability that a noncue word would be recalled was .380 for the one-cue test and .545 for the two-cue test. Clearly, recall rate improved significantly with more cues from the quartet $[t(14)=8.48, p<.001]$. All the models expect two-cue recall to exceed that for one-cue. The question to be decided is whether the two-cue recalls correspond to what the models predict in light of the one-cue results.

The most interesting results of this experiment concern the fit of the memory models to the data and the parameter changes between the two groups. The expected frequencies for the three models, summed within each group, are given in Table 1. Throughout this paper all theoretical fits were obtained by minimizing the chi-square using the STEPIT program (Chandler, 1969). We discuss each model in turn.

Horizontal model. The horizontal model was fit individually to each subject's one-cue and two-cue data. There were 3 degrees of freedom (df), since there were two parameters $(\theta$ and $\mathrm{p})$ and the seven recall frequencies had two sets that added to 40 . The model fit the data very well, with only one subject's fit yielding a significant discrepancy at the .05 level and two more at the .10 level. The overall chi-square, summed over subjects in both groups was very satisfactory $\left[\chi^{2}(48)=\right.$ $52.29, p>.20]$. The means of the estimated parameters are $\theta=.779$ and $p=.708$ for the informed subjects, and $\theta=.776$ and $p=.637$ for the control subjects. These estimates suggest that the informed group was more likely to encode items into the structure (higher $p$ value), but that the interitem connections were no stronger or more probable. However, neither $\theta$ nor $p$ differed significantly across the two groups [both $t(14) s<.449$ ], reflecting the failure of the experimental manipulation, as noted earlier.

Schema model. The schema model, with access and response parameters, was fit to the distributions of one-cue and two-cue recalls for each subject individually. This model fit the individual data exceedingly well, with only 1 subject of the 16 yielding a significant discrepancy. Summing the chi-square values for the individual fits (each with $3 \mathrm{df}$ ) yields a total chi-square of 39.07 on $48 \mathrm{df}$, which indicates a very good fit of the model. The mean parameter estimates were $a=.618$ and $\mathrm{r}=.680$ for the informed group and $\mathrm{a}=.531$ and $r=.654$ for the control group. Again, neither a nor $r$ differed significantly across groups [both $t(14) s<.514]$, though both parameter estimates were lower in the control group, as predicted. A more successful manipulation would probably have increased the schema-access parameter in the informed group but have had little effect on the response parameter.

Fragment model. The fragment model has four parameters to estimate from $5 \mathrm{df}$ in the data, leaving only $1 \mathrm{df}$ to test the fit. This model was fit both to the data of individual subjects and to pooled data of the two groups. Two subjects' fits were discrepant at the .05 level. Summed over subjects, the chi-square was 30.06 with $16 \mathrm{df}(\mathrm{p}<.05)$. For the fit to the pooled data, the chi-square was 5.29 with 1 df $(p<.05)$.

The parameter estimates obtained by fitting the 
Table 2

Fragment Estimates for Informed and Control Subjects for Experiment 1

\begin{tabular}{lcc}
$\begin{array}{c}\text { Fragment } \\
\text { Type }\end{array}$ & \multicolumn{2}{c}{ Estimated Probability } \\
Informed & Control \\
\hline 4 & .22 & .15 \\
3,1 & .35 & .33 \\
2,2 & .02 & .03 \\
$2,1,1$ & .22 & .22 \\
$1,1,1,1$ & .19 & .27 \\
\hline
\end{tabular}

individual subjects were similar to the ones derived from the group data, and the former set is displayed in Table 2. Two points should be noted. First, the probability of the multiple fragment $(2,2)$ was low in both groups. Only 6 of the 16 subjects had nonzero estimates of this fragment, and the fits to the groups' data yielded zero estimates for both groups. Second, the parameter changes from the control to the informed group are difficult to interpret. All fragment probabilities are the same across groups, except for gains in remember-all (4) compensated by losses in forget-all $(1,1,1,1)$. This pattern is not comprehensible to us.

This initial experiment, then, has provided some useful results. First, two cues were clearly stronger recall prompts than was one cue. Second, the horizontal and schema models provided an excellent account of this "additivity of memory cues," in that the same parameters fit the one-cue and two-cue results. With these quartets, the data were consistent with the idea that the combined prompting power of the two-cue package could be explained by "adding up" (in a special modeldetermined manner) the independent contributions of the prompting power of the two cues. There was no special "configural" information that emerged in the two-cue package.

We were pleased at how well the horizontal and schema models fit the data and interrelated the one-cue and two-cue data. The fragment model did not fare well in fitting the individual or group data. We were somewhat dismayed to find that the horizontal and schema models fit the data equally well, providing no preferred choice. This is surprising given the rather different appearances of the models. We had hoped that parameter estimates from the experimental manipulation, of informing subjects of a relevant schema for each quartet, would help to differentiate the models. Unfortunately, this manipulation was ineffective, since control subjects quickly discovered the underlying schemata anyway. Even so, informed subjects gave considerably more thematic intrusions than did controls. The next experiment tried again to differentiate the models.

\section{EXPERIMENT 2}

The second experiment was similar to the first, but provided a stronger test of the models by increasing the words per cluster from four to five. This increase, along with testing by one, two, or three cues, yields 12 rather than the former 7 frequencies to be predicted. Thus, the horizontal and schema models must fit nine independent frequencies with the same two parameters, ( $\theta$ and $p$ or $a$ and $r)$, while the fragment model will need six independent fragment parameters [for the seven fragments, $(5),(4,1),(3,2),(3,1,1),(2,2,1),(2,1,1,1)$, and $(1,1,1,1,1)]$.

Two further changes were made from Experiment 1. Rather than selecting items interassociated by some scenario, object, or event, the items were chosen to have some common sense impression (e.g., round, red, loud). This change was made both as a standard desire for generality and as a method to examine the influence of a slightly different type of schema mediator. Though each item was chosen for its association to its sense impression, it was generally not associated to the other items in the same sense-impression cluster.

The second change was to forego the informed vs. control comparison and to test all subjects in the informed procedure, with the sense impression announced as each quintet was shown. Though we still believe a manipulation of this type could be fruitful (see, e.g., Doll \& Lapinski, 1974), we were unable to construct a sufficiently large set of effective materials for which uninformed subjects could not infer the underlying structure.

\section{Method}

Materials. We constructed 72 critical quintets of words. First, some sense impression (e.g., round, soft, small) was chosen; then five words whose referents the authors judged to have this characteristic were assigned to this sense-impression quintet. The Underwood and Richardson (1956) norms were used insofar as possible to select many of the items. We avoided strong interword associates within a quintet. Two examples of sense impressions and their related word quintets are RED-ketchup, brick, robin, strawberry, firetruck; ROUNDhoop, pill, balloon, globe, donut.

The words were not systematically equated on concreteness, frequency, or association measures. A single word appeared in only one quintet. Some sense impressions were necessarily used more than once, but each use occurred in different learning lists. Each list contained 18 tested quintets plus a primacy buffer quintet presented at the front of each list but never tested. For each quintet, the five words were typed in a column, leftjustified, and a slide was made.

Each subject studied and was tested on all four lists in a random order. For each study list, 15 different cued recall test lists were composed, with each quintet cued five times with each of one, two, and three cues. Thus, each word appeared once as a single cue, twice as part of a double cue, and three times as part of a triple cue. For the double and triple cues, the other words in the cue were randomized within the constraints of the design.

Procedure. The procedure was similar to the informed condition of Experiment 1, with a few differences. Subjects were fully instructed about the procedure and asked to try to interrelate the five words using the sense impression announced by the experimenter at the presentation of each slide. The test sheets had 18 lines, with six cases each of one, two, or three cues per line and, correspondingly, four, three, or two spaces alongside for recall of the remaining words in the quintet. Subjects used the cardboard mask to cover every thing on the test sheet except current test cues. They were not allowed to return to earlier 
test cues. Subjects had 6 min to complete the 18 tests on each list. A short rest occurred after each test sheet.

Subjects. The subjects were 15 Stanford University students (ages 17-22 years), who were either paid volunteers $(n=8)$ or participating to fulfill a course requirement $(n=7)$. They were tested in small groups of one to four. Each session lasted about $1 \mathrm{~h}$.

\section{Results and Discussion}

The main results are the frequencies of recall patterns to one, two, or three cues. These are shown in Table 3 along with the predictions for the three models. The fit of each model is discussed in turn.

Horizontal model. The horizontal model should be fit only to individual data. Since, for a given subject, only 24 observations occurred in each cuing condition, it was necessary three times to combine two observation categories to insure that the expected frequencies were not below five. This pooling was done only within a given cue condition. The model was also fit to the data without pooling any categories, and the fit was just slightly worse. The model's fit to each subject's data has $4 \mathrm{df}$, that is, nine (independent frequencies) minus two (parameters) minus three (pooled cells).

Examining the horizontal model's fit to the 15 subjects, 5 showed a significant discrepancy at the .05 level and 4 more at the .10 level. Summing over subjects, $\chi^{2}(60)=150.31, p<.001$. The estimated parameters averaged $\theta=.853$ and $p=.622$. Besides the poor quantitative fit, 10 of the 15 subjects had $\theta$ estimates of 1.00 , which effectively reduces their model to a constrained fragment model. The predicted frequencies of the horizontal model are shown in Table 3; most of these predicted frequencies are quite discrepant from the observed frequencies.

Schema model. The schema model also must be fit to individual subjects, so similar collapsing of small-cell

Table 3

Observed and Predicted Pooled Frequencies of Recall Patterns for Experiment 2

\begin{tabular}{|c|c|c|c|c|}
\hline $\begin{array}{l}\text { Number } \\
\text { Recalled }\end{array}$ & $\begin{array}{l}\text { Observed } \\
\text { Frequency }\end{array}$ & $\begin{array}{l}\text { Hori- } \\
\text { zontal }\end{array}$ & Schema & $\begin{array}{l}\text { Frag- } \\
\text { ment }\end{array}$ \\
\hline \multicolumn{5}{|c|}{ One-Cue Testing Condition } \\
\hline 0 & 106 & 163 & 114 & 156 \\
\hline 1 & 63 & 39 & 70 & 56 \\
\hline 2 & 81 & 60 & 82 & 64 \\
\hline 3 & 56 & 61 & 64 & 43 \\
\hline 4 & 54 & 37 & 30 & 41 \\
\hline \multicolumn{5}{|c|}{ Two-Cue Testing Condition } \\
\hline 0 & 89 & 101 & 86 & 95 \\
\hline 1 & 113 & 83 & 114 & 102 \\
\hline 2 & 95 & 107 & 106 & 94 \\
\hline 3 & 63 & 69 & 54 & 69 \\
\hline \multicolumn{5}{|c|}{ Three-Cue Testing Condition } \\
\hline 0 & 126 & 101 & 113 & 95 \\
\hline 1 & 145 & 143 & 155 & 150 \\
\hline 2 & 89 & 116 & 92 & 115 \\
\hline
\end{tabular}

Note-All predicted frequencies are sums of predictions for individual subjects. frequencies was necessary. Three of the 15 subjects showed a significant discrepancy. Summing over subjects, $\chi^{2}(60)=86.51, p<.025$. However, this overall discrepancy was due to one subject whose chi-square was nearly twice as large as the next largest chi-square. Excluding this subject, the overall fit of the schema model was acceptable $\left[\chi^{2}(56)=67.54, p<.20\right]$. The parameter estimates, averaged over subjects, were $\mathrm{a}=.77$ and $\mathrm{r}=.48$. The predicted frequencies are shown in Table 3.

Fragment model. For the fragment model, there are seven possible fragments, so six parameters must be estimated, leaving $3 \mathrm{df}$. The seven fragments are $(5),(4,1),(3,2),(3,1,1),(2,2,1),(2,1,1,1)$, and $(1,1,1,1,1)$, and the corresponding estimated probabilities for the group data are $.11, .15, .03, .27, .03$, .31 , and .10 . The overall fit to the group data was poor $\left[\chi^{2}(3)=47.72, p<.001\right]$

The fragment model was also fitted to the data of individuals, without pooling cells. Six of the 15 subjects showed a significant discrepancy, with the summed chi-square indicating a significantly poor fit $\left[\chi^{2}(45)=\right.$ $124.29, \mathrm{p}<.001]$. Even excluding the two subjects showing the worst fits, the fragment model still failed significantly in fitting the data $\left[\chi^{2}(39)=86.03\right.$, $\mathrm{p}<.001]$.

The results of the second experiment, then, provide more support for the schema model than for the other two models. The horizontal model fared particularly badly in its predictions. Related evidence against the horizontal model comes from earlier research on intralist cuing, in which additional cues improve recall only if they access a previously unavailable organizational unit (Roediger, 1973; Slamecka, 1968; for a review, see Crowder, 1976, pp. 342-351).

For all three models, considerable mispredictions occurred in those cells of Table 3 involving total recall or complete failure of recall to one cue. The schema model did not fare as badly as the fragment model in these cells, with its worst misprediction being the probability of recalling four words given one cue. The fragment model consistently mispredicted these cells, with four of the six predictions off by 13 or more.

Part of the difficulty in fitting these data is that the average recall probability does not increase with the number of cues (one, two, or three) nearly as much as anticipated by all the models. The observed recall probability averaged .423 for the one-cue test, .456 for the two-cue test, and .449 for the three-cue test. To consider just the best-fitting example, the schema model predicts (averaged over individuals) that the average recall probabilities should be $.38, .45$, and .47 for one-, two-, and three-cue tests, respectively. (The expected recall probability with $C$ cues is $\mathrm{r}[1-(1-\mathrm{a}) \mathrm{C}]$, and for large a this rises quickly to $r$ as $C$ is increased.) In contrast to Experiment 2, the recall probabilities in Experiment 1 on one-cue and two-cue tests (.380 and $.545)$ were well predicted by the schema model $(.390$ 
and .543). Although the schema model is not significantly discrepant from the data of Experiment 2, the evidence for the model would clearly have been more compelling had the data revealed a strong "cue additivity" effect. The anticipated increase in recall with number of cues may be masked by a progressively inhibitory influence on retrieval of output interference (Roediger, 1973). Though output interference is not assumed by any of these models, such interference is believed to arise from a rule that prematurely halts retrieval attempts, rather than from the structure of the trace. ${ }^{2}$

\section{EXPERIMENT 3}

Though Jones (1976, 1978a, 1978b) has reported acceptable fits of several sets of data by his fragment model, our first two experiments yielded data that were not well fit by this model. Our third experiment was designed as a more direct test of the fragment theory. In particular, it investigated two basic assumptions of the fragment theory, viz., symmetry of associations and all-or-none recall (Jones, 1976, pp. 290-291). These two properties jointly imply that each item in a memory fragment has the same probability of activating that fragment and that all elements of an activated fragment can be produced as responses. These properties strongly predict consistent recall over a series of tests with the same cluster trace. To illustrate, suppose on a first test with the cluster $W, X, Y$, and $Z$ that $W$ is given as a cue to which $X$ and $Y$ are recalled. This fact implies that the subject has a three-element fragment for this quartet, $(W, X$, and $Y)$. Thus, giving $X$ or $Y$ as a cue on a later test of this quartet for the subject should evoke the other two members of the fragment, but it should never evoke $Z$. Similarly, providing $Z$ as a cue should produce no recall whatsoever, since it is supposedly not in the memory fragment remaining from the cluster. This example illustrates the strong predictions the fragment model delivers for certain sequential tests.

Such sequential test procedures have less discriminating power for the horizontal and schema models, even assuming that their links are available in an all-or-none rather than a probabilistic fashion, since these models posit distinct one-way associations between two elements. To illustrate, consider the schema model (refer to Figure $1 \mathrm{~b}$ ). If cue $\mathrm{W}$ produced responses $\mathrm{X}$ and $\mathrm{Y}$, this implies that $W$ has an a association into the schema, $X$ and $Y$ have $r$ associations from the schema, and $Z$ does not have an $r$ association from the schema. Cuing with $\mathrm{X}$ may produce $\mathrm{Y}$, or $\mathrm{W}$ and $\mathrm{Y}$, or nothing, depending on whether or not $W$ has an $r$ association and $X$ has an a association. The only strong prediction for this second test is that, if cue $X$ produces $Y$ or $W$, it should not produce $Z$ as well. Cuing with $Z$ may produce nothing, or $\mathrm{X}$ and $\mathrm{Y}$, or $\mathrm{W}, \mathrm{X}$, and $\mathrm{Y}$. The horizontal model for this sequential cuing procedure provides even fewer strong qualitative predictions.
After conducting this experiment, we discovered that Jones (1978a) had recognized these sequential implications of the fragment model and tested them. In his experiment, he presented subjects with many sets of nine slides to remember on immediate tests. Each slide had a different common object, each in a different color, which appeared in a different spatial location in a matrix. Subjects also had to remember the temporal position of the slides in the input list (first, second, etc.). Subjects were later cued with each feature (color, object, location, and temporal position) and had to choose the values of the three other features with which it had been shown. The temporal position variable yielded results different from those of the other three variables, so Jones analyzed it separately. Several comments are warranted concerning problems of the experiment, model fitting, and results.

During testing, Jones (1978a) equally spaced the four tests of a particular pattern (cuing with its different attributes). Though the test order was not confounded with the study order, this cyclic test information, if noticed, could increase the obtained symmetry. It is preferable to avoid such procedures. A second methodological point is that since only three features were used in the model analysis (temporal position having been eliminated), little leverage remained for strong tests of the model. For example, with three attributes, one is unable to observe the multiple fragment $(2,2)$ that is an important differentiating aspect of Jones' theory.

Jones posited three important alterations of his model which undercut its interesting, strong predictions. Three secondary mechanisms were postulated to operate upon the basic memory fragment: recall failure, forgetting, and guessing. Two types of recall failure were postulated for each feature. "First, a cue may fail to excite its appropriate fragment; second, although a fragment is excited, one of its components may fail to be produced as answer" (Jones, 1978a, p. 4). Note that these alterations rescind the strong consistency mentioned earlier as a prediction of the model. The model has been hedged so that qualitative predictions of recall patterns over successive cuings no longer provide strong tests; rather, the extended version of the fragment model can be tested only by its quantitative fit to such data. Though the strong version of the model accounted for only 533 of the 941 observations in Jones' experiment (of which 409 were all-or-none recall), the weakened version assuming recall failures, forgetting, and guessing parameters fit the data satisfactorily. However, the estimated recall failure parameters were small, at $5.15 \%$ and $1.86 \%$.

We used the sequential cuing procedure to collect data to test the fragment model. However, we should first note that two aspects of this procedure may be expected to yield overestimates of associative symmetry. First, repeated testing of the same memory cluster can produce learning of new pathways, which can lead on later tests to the appearance of symmetry. To illustrate 
with the schema model, suppose that item $\mathrm{W}$ has only an a association and $X$ and $Y$ have only $r$ associations. If $W$ is used as the first cue, $X$ and $Y$ will be recalled. Assuming that the subject can learn to associate the responses back to the cue, there is some probability that the $\mathrm{r}$ association to $\mathrm{W}$ and a associations from $\mathrm{X}$ and $\mathrm{Y}$ will then be established as a result of this test exposure. If so, a second cuing with $\mathrm{X}$ may now produce $\mathrm{W}$ and $\mathrm{Y}$, suggesting symmetry. However, if the order of the cuing had been $X$ and then $W$, recall would have been nothing to cue $\mathrm{X}$, and $\mathrm{Y}$ to cue $\mathrm{W}$, respectively, which provides no support to symmetry. This example illustrates that the sequential test procedure may overestimate symmetry. Second, the appearance of symmetry could arise spuriously, since recall of some items conditionalized on success or failure of recall of other items capitalizes on subject and item differences to produce bias (Runquist, 1973). Thus, for example, easy items may be very likely both to retrieve fragments and to evoke responses, whereas difficult items may do neither. These two factors may lead to an overall appearance of symmetry for pooled data without symmetry being true of individ. ual clusters for individual subjects.

Setting aside these complications, our third experiment attempted to test the fragment model by sequential cuing. The equally spaced test intervals (of Jones, 1978a) have been avoided, but the two sources of symmetry mentioned above seem unavoidable. The findings are interpreted with these in mind.

\section{Method}

Design. Subjects had one study-recall cycle on each of four lists of 12 quartets of unrelated words. Each quartet was cued twice during the test, each time with a different single word.

Materials. We constructed 48 quartets of words by randomly selecting 192 of the 360 words used in Experiment 2. Any quartet that contained two or more highly related words was rejected, and a new quartet drawn. The 48 quartets were randomly divided into four lists of 12 . A primacy buffer quartet was presented at the front of each list but was not tested. For each quartet, the four words were typed on successive lines, left-justified, and slides were made.

Each subject studied and recalled all four lists in a random order. For each list, we constructed 24 cued recall tests, 2 replications of each of the 12 possible test pairs for each quartet. With 18 subjects, only $1 \frac{1}{2}$ test sets could be used. The two tests of each quartet were ordered so that subjects could not use the input and output order of the quartets as a recall cue. The tests also exhibited a nearly constant interval between a quartet's study and its first test and between its two tests. To achieve these goals, the 24 tests were ordered as follows: The first 8 tests probed recall of the first eight quartets; the second 8 tests probed recall of the last four quartets and contained second tests probing for recall of the first four quartets; the last 8 tests were second tests for the last eight quartets. The 8 tests within each of the three test blocks were randomly ordered.

Procedure. The subjects were fully informed of the learning task and of the cued recall tests and were told that quartets could be tested more than once. They were told to study the word quartets so that, given one word, they could recall the other three.

The study and test procedure was similar to that for the control group in Experiment 1. The 13 slides were shown for $10 \mathrm{sec}$ each. For cued recall, the subjects were allowed $8 \mathrm{~min}$ at their own pace to complete the 24 tests. They were not allowed to go back to earlier tests. A short rest was given after each list.

Subjects. The subjects were 18 Stanford University students (ages 17-24 years) who either were paid $(\$ 2.50)$ volunteers $(n=3)$ or were fulfilling a course requirement $(n=15)$. They were tested in groups of one to four. The sessions lasted about $1 \mathrm{~h}$. The data from two extra subjects were excluded when an error was discovered in the program for counterbalancing the design.

\section{Results and Discussion}

Table 4 contains the observed frequencies of the 40 possible events. Each of the 18 subjects contributed 48 observations (pairs of cues), for a total of 864 . The notation labels the unordered quartet of words as $\mathrm{W}, \mathrm{X}$, $\mathrm{Y}$, and $\mathrm{Z}$, where $\mathrm{W}$ is the word used as the first test cue and $X$ is the word used later as the second cue. Thus, line 2 denotes the joint event when the first cue $W$ prompted recall of the three other words, but the second cue $X$ led to only two words, neither of which was word $\mathrm{W}$, which had been given as the first cue. Similarly, line 13 denotes the joint event when the first cue prompted recall of two words (X and $\mathrm{Y})$; then, when one of these words was given as the second cue, the only word recalled $(\mathrm{Z})$ was the one that had not occurred as either cue or response on the first test. Note that $Y$ and $Z$ are differentiated only when necessary, such as in the event of line 13 , but not in events such as line 15 .

Two features of the data should be noted before discussing the fits of the models' predictions. First, there was no retention loss between tests; the proportion of items recalled on the second test (.472) was slightly higher than that on the first test (.452). This result may be due to either no forgetting or extra learning on the first test trial, as mentioned earlier. The second aspect of the data to be noted is the large frequencies of total recall or total failure of recall, amounting to 522 of the 864 observations.

Fragment model. The main purpose of this study was to examine how well the fragment model's predictions fit the sequential cuing data. Its predicted frequencies may be compared with the data in Table 4 .

Six parameters were used in fitting the augmented fragment model: four fragment probabilities (with a fifth equaling one minus the other four) and two recall failure probabilities: $A$, the probability that a cue failed to activate a memory fragment that contained it, and $\mathrm{B}$, the probability that an item was not given as a response from an activated fragment that contained it. A particular pattern of recall on a cue pair (i.e., a line in Table 4) may usually occur from any of several underlying fragments. The derivation of these likelihoods is tediously lengthy and can be obtained by writing to the authors.

The six parameters were estimated from the 40 frequencies in Table 4 by minimizing the chi-square goodness of fit. The estimation was performed first on all 40 cells and again by collapsing low-frequency cells so that all expected cell frequencies were at least five. 
Table 4

Observed Recall Frequencies for Experiment 3, Predicted Frequencies of the Augmented Fragment Model, and Patterns Allowed by the Models

\begin{tabular}{|c|c|c|c|c|c|c|c|}
\hline & \multicolumn{2}{|c|}{ Recall Pattern } & \multirow[b]{2}{*}{ Observed } & \multirow[b]{2}{*}{$\begin{array}{l}\text { Fragment } \\
\text { Prediction } \\
\end{array}$} & \multirow[b]{2}{*}{$\begin{array}{c}\text { Hori- } \\
\text { zontal }\end{array}$} & \multirow[b]{2}{*}{ Schema } & \multirow[b]{2}{*}{$\begin{array}{l}\text { Frag- } \\
\text { ment } \dagger\end{array}$} \\
\hline & $\begin{array}{c}\text { Recall to } \\
\text { W Cue }\end{array}$ & $\begin{array}{c}\text { Recall to } \\
\text { X Cue }\end{array}$ & & & & & \\
\hline 1. & $\mathrm{XYZ}$ & WYZ & 224 & 151.5 & + & + & + \\
\hline 2. & & YZ & 3 & 16.2 & + & + & \\
\hline 3. & & WY* & 3 & 32.4 & & & \\
\hline 4. & & W & 1 & 1.7 & & & \\
\hline 5. & & $\mathrm{Y}^{*}$ & 2 & 3.5 & + & & \\
\hline 6. & & 0 & 1 & 8.9 & + & + & \\
\hline 7. & $\mathrm{XY}^{*}$ & WYZ & 5 & 32.4 & & & \\
\hline 8. & & $\mathrm{YZ}$ & 1 & 3.5 & & & \\
\hline 9. & & WY & 64 & 53.1 & + & + & + \\
\hline 10. & & WZ & 1 & 3.5 & & & \\
\hline 11. & & W & 5 & 5.7 & & & \\
\hline 12. & & $\mathrm{Y}$ & 1 & 5.7 & + & + & \\
\hline 13. & & Z & 0 & .4 & & & \\
\hline 14. & & 0 & 3 & 5.0 & + & + & \\
\hline 15. & $\mathrm{YZ}$ & WYZ & 17 & 16.2 & + & + & \\
\hline 16. & & $W^{*}$ & 1 & 3.5 & & & \\
\hline 17. & & $\mathrm{YZ}$ & 3 & 1.7 & + & + & \\
\hline 18. & & W & 0 & .2 & & & \\
\hline 19. & & $\mathrm{Y}^{*}$ & 0 & .4 & + & & \\
\hline 20. & & 0 & 14 & 32.7 & + & + & + \\
\hline 21. & $\mathrm{X}$ & WYZ & 0 & 1.7 & & & \\
\hline 22. & & WY* $^{*}$ & 1 & 5.7 & & & \\
\hline 23. & & $\mathrm{YZ}$ & 0 & .2 & & & \\
\hline 24. & & W & 33 & 27.4 & + & + & + \\
\hline 25. & & $\mathrm{Y}^{*}$ & 1 & .6 & & & \\
\hline 26. & & 0 & 6 & 4.6 & + & + & \\
\hline 27. & $\mathrm{Y}^{*}$ & WYZ & 5 & 3.5 & + & & \\
\hline 28. & & $\mathrm{YZ}$ & 2 & .4 & + & & \\
\hline 29. & & WY & 13 & 5.7 & + & + & \\
\hline 30. & & WZ & 1 & .4 & & & \\
\hline 31. & & $\mathrm{~W}$ & 0 & .6 & & & \\
\hline 32. & & $\mathbf{Y}$ & 6 & .6 & + & + & \\
\hline 33. & & $\bar{Z}$ & 12 & 19.5 & + & & + \\
\hline 34. & & 0 & 53 & 49.9 & + & + & + \\
\hline 35. & 0 & WYZ & 7 & 8.9 & + & + & \\
\hline 36. & & WY* & 9 & 5.0 & + & + & \\
\hline 37. & & $\mathrm{YZ}$ & 13 & 32.7 & + & + & + \\
\hline 38. & & W & 19 & 4.6 & + & + & $T$ \\
\hline 39. & & $\mathrm{Y}^{*}$ & 36 & 49.9 & + & + & + \\
\hline \multirow[t]{2}{*}{40.} & & 0 & 298 & 263.9 & + & + & + \\
\hline & & Total & 864 & 864.0 & & & \\
\hline
\end{tabular}

Note-The predicted frequencies were fit by collapsing 13 cells. recall pattern. TStrong form of fragment model.

*These are cells for which $Y$ and $Z$ are not differentiated by the

The parameter estimates and poorness of fit were roughly equivalent for both minimizations, so the reported fit is for the collapsed cells. The chi-square showed a highly significant discrepancy $\left[\chi^{2}(20)=\right.$ $238.04, p<.001]$. The estimated probabilities for the fragments of type $(4),(3,1),(2,2),(2,1,1)$, and $(1,1,1,1)$ were $.35, .19, .04, .16$, and .26 , respectively. The estimates for the recall failure probabilities were .04 that a cue would fail to excite a fragment that contained it (A), and .10 that a response would not be given from an activated fragment that contained it (B).

Comparing the observed and predicted frequencies is informative. First, the only recall patterns expected by the strong fragment theory are those in lines $1,9,20$,
$24,33,34,37,39$, and 40 ; these contain $86.5 \%$ of the 864 observations. However, we note that 522 of the 864 observations were either total recall or complete failures to both cues; these events are unrevealing since most models will predict them. Of the remaining 342 observations, 117 , or $34.2 \%$, occur in patterns that are prohibited by the strong version of the fragment model. Thus, by focusing on the discriminating data, the strong fragment model can be decisively rejected. A second observation regards the recall patterns that in theory are theoretically possible only if all items are in the memory trace (lines $1-8,10,13,15-19,21,23,27,28$, 30 , and 35 ). While the total recall (line 1 ) is greatly underpredicted, most other events in this subset are 
overpredicted. In particular, those patterns of recall possible from a (4) fragment with one recall failure of a B type (lines 2, 3, 7, and 15) show strong overpredictions (observed 28 vs. predicted 97.2).

Horizontal and schema models. Though this study was not designed to test the horizontal and schema models, some simple analyses can be examined. Since the models must be fit to individual subjects, there are not enough data points to allow a thoroughly reliable analysis.

As mentioned before, the strong forms of the horizontal and schema models, assuming deterministic link learning and without recall failures of the type proposed for the weakened fragment theory, predict that certain patterns of sequential recall should not occur. For example, in the schema model, if $\mathrm{W}$ leads to $\mathrm{X}$ and $\mathrm{Y}$, then the a link exists for $W$, and the $r$ links exist for $X$ and $Y$ but not for $Z$. Thus, a subsequent test with cue $X$ may lead to nothing, or to $Y$, and possibly to $W$ but not to $Z$. A fit of these predictions for each subject is impossible without many more observations. However, since each model predicts that certain sequential events should not occur, one may examine the proportion of observations in these disallowed cells. The recall patterns allowed by each model are marked to the right in Table 4 . Of the 40 cells, 20 are contrary to the schema model predictions. However, the 20 disallowed cells contain only $4.7 \%$ of the observations. Thus, the qualitative pattern of recall is not particularly discrepant from the schema model.

The horizontal model also predicts the qualitative pattern quite well. This model allows 25 of the 40 possible patterns. The 15 patterns not allowed contain $2.3 \%$ of the observations.

A quantitative analysis of individual subjects may be performed on a much reduced form of the data. One may ignore the sequential testing and examine the probability of recalling certain numbers of words (zero, one, two, or three), given one cue. This analysis was performed separately for the first and second tests for each subject. The resulting chi-square has $1 \mathrm{df}$ (three independent data points minus two parameters). For the schema model, the fits, summed over subjects, yield highly significant discrepancies, with chi-squares of $61.54(18 \mathrm{df})$ for the first test and 43.67 for the second test (both ps $<.001$ ). Of the 18 subjects, 7 showed significantly discrepant fits on the first test and 6 on the second test. The parameters were roughly equivalent and changed little between the two tests. The parameters a and $\mathrm{r}$ averaged .612 and .635 for the first test and .616 and .653 for the second test. Collapsing over test trials, the observed frequencies for zero, one, two, and three correct recalls were 753,249 , 232 , and 494, respectively, while the corresponding predicted frequencies were $731,192,343$, and 462 . Hence, the partial recalls (one and two words) were poorly predicted.

The horizontal model did slightly better in fitting these data. For the results of the first test, five subjects showed significant discrepancies, and the total chisquare was 48.11 with $16 \mathrm{df}(\mathrm{p}<.001)$. For the second test, however, no subjects showed significant discrepancies $\left[\chi^{2}(16)=27.37, p>.05\right]$. The mean parameter estimates for the first test were $\theta=.666$ and $p=.718$, and for the second test were $\theta=.548$ and $p=.779$. The parameters did not differ significantly between tests [both $t(34) s<.706$ ]. The predicted frequencies were $730,238,297$, and 463 , which are closer to the partial recall frequencies than the schema model, though still not very close.

This third experiment, then, provides differing pieces of evidence against the fragment model, the schema model, and the horizontal model. The fragment model was tested most rigorously by having to account quantitatively for all 40 data points. A significant discrepancy under such conditions should not be interpreted as causing total rejection of the fragment model for all experiments, particularly in light of the success Jones $(1976,1978 \mathrm{a}, 1978 \mathrm{~b})$ has had in fitting this model.

Three problems, however, seriously question the viability of the fragment model. First, its misfit to the data from this third experiment was not only statistically significant, it was extremely poor. A second difficulty is that, once again, the probability of the multiple fragment trace $(2,2)$, an important differentiating feature of the fragment model, was very small (.04). Third, this experiment has not just examined the fit of quantitative predictions, as did the first two experiments and Jones' (1976, 1978b), but has investigated directly two basic tenets of the fragment model, namely, symmetry of associations and all-or-none recall. Assuming that cuing and recall from a fragment may fail, the augmented fragment model can then qualitatively expect any pattern of sequential recall. However, despite this increased flexibility as well as the test biases towards symmetry mentioned earlier, the quantitative fit of the theory was extremely poor.

The schema and horizontal model provided fair qualitative accounts of the data, but experienced difficulty quantitatively fitting the marginal recall probabilities. This finding was rather surprising after the first two experiments. More is said on this point in the discussion.

\section{GENERAL DISCUSSION}

The results of these three experiments, though not conclusive, yield some interesting results for models of associative recall. Foremost, the fragment model did poorly in every experiment. Its predictions for recall of related word clusters were unsatisfactory, and the results of the third experiment with unrelated words cast doubt on its major assumptions. We also find problematic the small probabilities estimated for multiple fragments, which have been consistently in the range of .00 to .05 .

The horizontal and schema models both satisfactorily fit the data from the first experiment, and their parameters changed with conditions in an interpretable manner. 
For the second experiment, the horizontal model did not provide an adequate account of the data, while the schema model fared well. In the third experiment, both models provided adequate qualitative accounts, but their quantitative predictions of one-cue recalls were not satisfactory.

Before ascribing the difficulty in the third experiment to the use of unrelated materials, the results of Arnold (1976) should be noted. In several experiments, Arnold's subjects learned unrelated triplets of words, with memory assessed by one- or two-cue recall and recognition of pairs and/or triplets. He found that a model equivalent to the schema model gave satisfactory accounts of the data. The theoretical formulation to follow provides a possible account of the misfit of this model in our third experiment.

In the following, we discuss a framework for viewing the influence of preexperimental knowledge on the structure of the memory trace in terms of spreading activation and strengthening of associations. We then examine how the schema model is related to other models of memory structure.

\section{A Spreading Activation Interpretation}

The framework we propose for examining the influence of preexperimental knowledge is most easily explained by reference to a network representation of semantic knowledge. Memory is considered to be a set of propositions interconnecting concepts (nodes), with the relations between concepts specified by the connection type (this representation is described in detail by J. R. Anderson, 1976). When a word is presented, the concept to which it refers is activated and the activation spreads in a parallel limited-capacity manner to the other concepts to which it is connected. As the spreading continues over time, the activation from the two sources may intersect at one or more concept nodes. For example, "predatory birds" might be an intersection concept for "hawk" and "buzzard." Two learning assumptions are needed to convert this spreading activation idea into a memory model. First, when an intersection concept (S) connecting concepts $\mathrm{X}$ and $\mathrm{Y}$ is found, the pathways involved are strengthened, that is, the links from concepts $X$ and $Y$ to intersection concept $S$ as well as the reverse links are strengthened. Second, the strengthened paths through $S$ are likely to be used to recall $\mathrm{Y}$ when the person is later cued with $\mathrm{X}$, or vice versa.

Two features of this framework are noteworthy. First, presented concepts will vary in their ability to activate ("cue") the mediating concept, depending upon how strong are the links to the mediating concept relative to the other links from this cuing concept. Similarly, the mediating concept, once activated, will be more or less likely to activate a given concept ("response") dependent on the strength of that connection relative to the other connections from the mediating concept. Thus, using an earlier example, "chair" may not be very likely to activate the haircut concept, but if some concept did activate the haircut concept (e.g., scissors), "chair" may be likely to be given as a response.

A second point about this general framework is that it allows various subgroups among concepts even for a given cluster. That is, depending upon prior knowledge structures, the number of concepts associated through an intersection will vary. Though some clusters (e.g., the scripts of Schank \& Abelson, 1977) would be represented by a set of strongly interconnected propositions, such that the presentation of any one of the concepts is likely to activate the whole structure, many clusters would not. For a given cluster, the mediating concept activated (through intersection) may vary greatly over time, depending upon factors such as semantic focus and context. Also, intersections (particularly across clusters) may associate or chunk together differing numbers of items.

The general model from this framework is one in which the memory trace incorporates the items through a mediating concept. The span of the mediating concept is strongly influenced by the preexperimental associations of the items. The horizontal and schema models are special cases, differing on the span of the mediating concept. The horizontal model arises in cases in which all intersections (subgroups) include only two items, each item is grouped with every other, and all connections are approximately the same distance (i.e., equal numbers and strengths of intervening connections). The schema model arises when a single mediating concept occurs at an intersection containing all of the items. Thus, the horizontal and schema models differ according to the span of the intersecting concept used. With appropriate normative data, the span of the intersection could be varied across materials. The schema model would mispredict the data of Experiment 3 if the span of the intersecting concept for a word quartet varied from two to four across quartets.

\section{Related Theories}

In this last section, we focus on the schema model because of its superior fit to the data and its close relation to other current models of memory structure. First, the schema model seems an appropriate rendering of Wickelgren's (1979) theory wherein elements within a group become interconnected by virtue of their vertical associations to a higher order node. He refers to the higher order unit as a "chunk node," and its role is to provide access from one element to another within an associative complex. Moreover, Wickelgren proposed a physiological intersection process, and a pathway strengthening process similar to that proposed here. Second, a closely similar theory is Estes' (1972) model of associative coding. He assumed that cognitive units that are grouped together become chunked together via vertical associations to a "control unit," that each elementary unit in the proper context may activate the control unit, and that its activation can make other 
elements (subsumed by that control unit) available as responses. Identifying the central hub of the schema model with the control unit of Estes' coding model brings out the essential similarity of the two theories.

Third, the schema model is viewed as a simplified version of the associative networks envisioned by semantic models such as HAM (J.R. Anderson \& Bower, 1973), ACT (J. R. Anderson, 1976), ELINOR (Norman et al., 1975), and the predicate calculus representation used by Kintsch (1974). In these models, the elements connected are semantic concepts that are retrieved by words; the pathway connecting any two items may be several links long, each link labeled with some functional distinction (e.g., subject, instrument). In the general schema model, the labels are disregarded and multilink chains are summarized by a single link with a single parameter (its "success" probability) attached to the chain as a whole. In these terms, the interpretation of the central hub of the schema model is that it is the proposition, or propositional elaborations, that the learner has used to interrelate and memorize the group of items. Network theories assume particular connectivities and distances between concepts in a proposition, and, if desired, the theorist can represent the varying internode distances by different $a$ and $r$ parameters in the schema model.

Fourth, our schema model is closely related to the ideas of frames (Minsky, 1975), schema (R.C. Anderson, 1977; Rumelhart \& Ortony, 1977), and scripts (Schank \& Abelson, 1977). A schema is a large conceptual structure preexisting in memory; it interrelates a number of actors, objects, and properties; it gives general information about these categories along with the type of values which fill those variable slots; it can be applied to new cases by instantiating the variables in terms of the value of particular objects or events in the case at hand. Of relevance to our materials for Experiment 1 are schema for activities (camping, cutting hair) or situations (chemistry lab, dentist's office). Thus, when the person reads "test tube," "litmus paper," "Bunsen burner," and "acid," by an intersection search in memory, the relevant "chemistry lab" schema is activated. These items are already interassociated props or objects within a scripted activity familiar to our subjects. Given the schema at the intersection, learning then consisted, first, of strengthening each word-to-schema and schema-to-word linkage, and, second, of tagging specifically the presented items of the schema as having occurred in the list studied (rather than "bench," "pipette," etc.). These learning functions are reflected in the access parameter and the response parameter in the mathematical model. If schema access succeeds but the subject fails to discriminate accurately between the mentioned vs. unmentioned props of the script, then script-related words are likely to intrude in recall.

Much theoretical debate has occurred over the most efficient or proper way to represent the structural relationships among concepts in a proposition, schema, or script. However, it has proved difficult to obtain unequivocal evidence in favor of particular proposals about the structure or labeling of basic semantic relations within these representations (for a review of the difficulties, see J. R. Anderson, 1976, chap. 8). The schema model presented here is neutral on these debatable issues, summarizing the series of connections by the probability of success. Consequently, the model may be recommended for use by investigators working on associative learning but wishing to avoid the strong commitment to specific structural representations of memories made by the extant theories of the day.

\section{REFERENCE NOTE}

1. Goetz, E. T., Anderson, R. C., \& Schallert, D. L. The representation of sentences in memory (Tech. Rep. 144). Urbana: University of Illinois, Center for the Study of Reading, 1979.

\section{REFERENCES}

Anderson, J. A. Neural models with cognitive implications. In D. LaBerge \& S. J. Samuels (Eds.), Basic processes in reading: Perception and comprehension. Hillsdale, N.J: Erlbaum, 1977.

Anderson, J. R. Language, memory, and thought. Hillsdale, N.J: Erlbaum, 1976.

Anderson, J. R., \& Bower, G. H. Human associative memory. Washington, D.C: Winston, 1973.

Anderson, J. R., \& Bower, G. H. Human associative memory (rev. ed.). Hillsdale, N.J: Erlbaum, 1980.

Anderson, R. C. The notion of schemata and the educational enterprise. In R. C. Anderson, R. J. Spiro, \& W. E. Montague (Eds.), Schooling and the acquisition of knowledge. Hillsdale, N.J: Erlbaum, 1977.

Arnold, P. On the nature of associations. Unpublished doctoral dissertation, Stanford University, 1976.

Asch, S. E., \& Ebenholtz, S. M. The principle of associative symmetry. Proceedings of the American Dhilosophical Society, 1962, 106, 135-163.

BruCE, D. Single probes, double probes, and the structure of memory traces. Journal of Experimental Psychology: Human Learning and Memory, 1980, 6, 276-292.

Chandter, J. P. STEPIT-Finds local minima of a smooth function of several parameters. Behavioral Sicence, 1969, 41, 81-82.

Crowder, R. G. Principles of learning and memory. Hillsdale, N.J: Erlbaum, 1976.

Doll, T. J., \& LAPINSKi, R. H. Context effects in speeded comprehension and recall of sentences. Bulletin of the Psychonomic Society, 1974, 3, 342-344.

Estes, W. K. An associative basis for coding and organization in memory. In A. W. Melton \& E. Martin (Eds.), Coding processes in human memory. Washington, D.C: Winston, 1972.

JoNes, G. V. A fragmentation hypothesis of memory: Cued recall of pictures and of sequential position. Journal of Experimental Psychology: General, 1976, 105, 277-293.

JoNEs, G. V. Repeated cueing and the structure of recall. British Journal of Mathematical and Statistical Psychology, 1978, 31, 1-10. (a)

Jones, G. V. Tests of a structural theory of the memory trace. British Journal of Psychology, 1978, 69, 351-368. (b)

$\mathrm{K}_{\mathrm{INTSCH}}, \mathrm{W}$. The representation of meaning in memory. Hillsdale, N.J: Erlbaum, 1974.

KoHonen, T. Associative memory: A system-theoretic approach. Berlin: Springer-Verlag, 1977.

Minsky, M. A framework for representing knowledge. In P. H. Winston (Ed.), The psychology of computer vision. New York: McGraw-Hill, 1975. 
Norman, D. A., Rumelhatt, D. E., \& The LNR Research Group. Explorations in cognition. San Francisco: Freeman, 1975.

ROEDIGER, H. L. Inhibition in recall from cueing with recall targets. Journal of Verbal Learning and Verbal Behavior, 1973, 12, 644-657.

Rumelhart, D. E., \& Ortony, A. The representation of knowledge in memory. In R. C. Anderson, R. J. Spiro, \& W. E. Montague (Eds.), Schooling and the acquisition of knowledge. Hillsdale, N.J: Erlbaum, 1977.

Runquist, W. N. Conditional recall and bias in memory. American Journal of Psychology, 1973, 86, 351-359.

Schank, R., \& Abelson, R. Scripts, plans, goals, and understanding. Hillsdale, N.J: Erlbaum, 1977.

Slamecka, N. J. An examination of trace storage in free recall. Journal of Experimental Psychology, 1968, 76, 504-513.

UNDERWOOD, B. J., \& Richardson, J. Some verbal materials for the study of concept formation. Psychological Bulletin, $1956,53,84-95$.

WiCKe LGREN, W. Chunking and consolidation: A theoretical synthesis of semantic networks, configuring in conditioning, S-R versus cognitive learning, normal forgetting, the amnesic syndrome, and the hippocampal arousal system. Psychological Review, 1979, 86, 44-60.

Wolford, G. Function of distinct associations for paired-associate performance. Psychological Review, 1971, 78, 303-313.

\section{NOTES}

1. Bruce (1980) investigated possible combination rules of cues. Though he interprets his results in terms of the fragment model (to be explained later), he acknowledges that they are consistent with many associative models, including one equivalent to the schema model (p. 290).

2. A possible change to incorporate output interference would be to redefine the r parameter as the probability that the schema-to-item connection is intact, rather than as the probability of response. We briefly investigated one model of this type in which the noncues (with intact $\mathrm{r}$ connections) and the cues were randomly sampled with replacement for $n$ "draws." The probabilities of the different response patterns are still a product of the probabilities of accessing the schema and the probabilities of responding given an activated schema, but the latter probabilities now depend on competition in sampling. We have not tried to systematically fit this model to the data, but informal fits (using the a and $\mathrm{r}$ estimates from the simple schema model) indicate that this sampling model can produce the slight nonmonotonicity of the results. For example, with $n=6$, recall probability estimates for one, two, and three cues are $.30, .38$, and .37 , respectively.

(Received for publication May 9, 1980; revision accepted July 3,1980 .) 